\title{
ANÁLISE DA RELAÇÃO ENTRE IMPORTAÇÕES E PRODUTIVIDADE: EVIDÊNCIA EMPÍRICA PARA A INDÚSTRIA DE TRANSFORMAÇÃO DO BRASIL*
}

\author{
Igor Ézio Maciel Silva ${ }^{1}$ \\ Jocildo Fernandes Bezerra ${ }^{2}$ \\ Ricardo Chaves Lima
}

RESUMO: A década de 1990 foi marcada por mudanças na economia brasileira, dentre elas a abertura comercial e a retomada do crescimento da produtividade. Muitas teorias e trabalhos empíricos apontam os impactos do comércio externo sobre a produtividade e o crescimento econômico, mas ainda não há consenso sobre essas questões. Este trabalho tem por objetivo examinar a relação entre o volume das importações e a produtividade do trabalho da indústria de transformação do Brasil. Para tanto, foram utilizados dados trimestrais das variáveis e a técnica dos vetores autorregressivos. Os resultados indicaram que o crescimento das importações influencia positivamente a produtividade.

PALAVRAS-CHAVE: Importações; produtividade; indústria de transformação; vetores autorregressivos.

CLASSIFICAÇÃO JEL: F10; O40; L60; C32.

\footnotetext{
*Artigo recebido em 23/09/2011 e aprovado em 17/03/2012.

${ }^{1}$ Doutorando do Programa de Pós-graduação em Economia da Universidade Federal de Pernambuco/ PIMES. Contato: igormacielsilva@gmail.com.

${ }^{2}$ Doutor em Economia pela Universidade de São Paulo e professor do Departamento de Economia da Universidade Federal de Pernambuco e do PIMES/UFPE. Contato: jocildo.bezerra@gmail.com.

${ }^{3}$ Doutor pela Universidade do Tennessee (EUA) e professor do Departamento de Economia da Universidade Federal de Pernambuco. Contato: chaveslima@gmail.com.
} 


\section{ANALYSIS OF THE RELATION BETWEEN IMPORTS AND PRODUCTIVITY: EMPIRICAL EVIDENCE FOR BRAZILIAN MANUFACTURING INDUSTRY}

ABSTRACT: The 1990s was marked by changes in the Brazilian economy, among them the revival of trade liberalization and productivity growth. Many theories and empirical studies show the impact of foreign trade on productivity and economic growth, but there is no consensus on those issues. This paper aims at examining the relationship between the volume of imports and labor productivity of Brazilian manufacturing industry, by using quarterly data for the variables and the vector autoregressive model. The results indicate that the growth of imports positively influences productivity.

KEYWORDS: Imports; productivity; manufacturing industry; vector autoregressive model. 


\section{INTRODUÇÃO}

No começo da década de 1990, o Brasil passou por profundas mudanças, destacando-se, entre outras, a abertura ao comércio externo, o Plano Real e as privatizações. A liberalização do comércio foi marcada pela redução do protecionismo à indústria nacional.

Até o final da década de 1980, as barreiras comerciais foram utilizadas como importante instrumento para garantir a estratégia de substituição de importações ${ }^{4}$ no Brasil. Nesse período, acreditava-se que a industrialização nos países não desenvolvidos seria possível, apenas, com proteção à indústria nascente e superávits na balança comercial, estes como forma de evitar a escassez de divisas. Assim, entre 1957 e 1988, a estrutura de proteção na economia brasileira se caracterizou por elevadas tarifas, barreiras não tarifárias, e regimes de importações (Averbug, 1999). O resultado foi uma reduzida penetração dos produtos estrangeiros e viés antiexportador (Bonelli e Pinheiro, 2008).

No final dos anos 1980, foi introduzida uma ampla reforma na política comercial que removeu a maior parte do sistema de proteção não tarifária, e reduziu gradativamente as tarifas para cerca de $1 / 4$ do que prevalecia no início daquela década (Arbache e Corseuil, 2001).

Há estudos indicando que a abertura comercial apresentou resultados favoráveis para a economia brasileira ${ }^{5}$. Dentre os benefícios, destacam-se: a contribuição para estabilização dos preços, crescimento das exportações, aumentos de salários e ganhos de produtividade. Após a reforma comercial, as receitas de exportações aumentaram tanto por causa de crescimento do volume, quanto pelo preço médio que se elevou durante todo o período entre 1991 a 2008. O crescimento do valor exportado foi expressivo entre 2003 e 2008, e menos vigoroso entre 1995 a $1999^{6}$ - período em que o câmbio esteve sobrevalorizado.

Existe um consenso de que o início da década de 1990 se caracteriza por uma ruptura na tendência de queda da taxa de crescimento da produtividade no Brasil, mudando o cenário em que altas taxas de crescimento do PIB eram conseguidas mais por causa do aumento na dotação de fatores do que por elevação de produtividade, sobretudo em alguns dos anos do período protecionista (Rossi Jr. e Ferreira, 1999).

\footnotetext{
${ }^{4}$ Política desenvolvida por Prebisch e Singer (1950) apud Silva (2004).

${ }^{5}$ Por exemplo: Bonelli et al. (1997), Bonelli e Fonseca (1998), Rossi Jr. e Ferreira (1999) e Arbache e Corseuil (2001).

${ }^{6}$ De acordo com dados do Ministério do Desenvolvimento, Indústria e Comércio Exterior (MDIC).
} 
A indústria de transformação do país, em particular, também volta a apresentar elevado crescimento da produtividade durante parte da década de 1990. Argumenta$-\mathrm{se}^{7}$ que o processo de abertura comercial teve um papel nesse fenômeno, pois aumentou a capacidade de aquisição de melhores insumos e bens de capital, intensificou a competição interna e elevou a competitividade dos produtos industriais, contribuindo para o acréscimo das exportações.

Apesar de ser possível identificar mudanças positivas após a abertura comercial, não existe um consenso em relação a todos os seus benefícios, ou, ainda, à forma pela qual o comércio influencia a economia e, em especial, a produtividade e o crescimento. De acordo com Bonelli e Pinheiro (2008), o foco da discordância se situa, especialmente, na direção da causalidade entre os dois processos.

A discussão a respeito do impacto do comércio internacional sobre a atividade econômica é antiga, datando de Adam Smith e sua teoria das "Vantagens Absolutas". Desde então, a teoria tem se desenvolvido. O pensamento neoclássico supera a ideia da necessidade de fatores ociosos, avança para a tese da realocação dos fatores, e alcança o estágio mais avançado dos efeitos dinâmicos em que há mudanças nas funções de produção e, portanto, contempla o conceito de crescimento da produtividade a partir de fatores do lado da oferta (Bezerra e Lima, 2005).

A teoria do crescimento endógeno, utilizando-se de modelos de equilíbrio geral, destaca o papel das mudanças tecnológicas. Grossman e Helpman (1991), Romer (1990) e (Afonso, 2001) concluem que o comércio internacional contribui para a elevação da taxa de crescimento da produtividade, porque promove o acesso a novos, e melhores insumos e tecnologias.

Nesse contexto, o presente trabalho objetiva examinar a relação entre o volume das importações e a produtividade do trabalho da indústria de transformação do Brasil. Para tanto, foi utilizada a análise de séries temporais - testes de causalidade de Granger, análise de cointegração e modelos de vetores autorregressivos com correção de erro.

$\mathrm{O}$ estudo constitui-se de cinco partes, incluindo a presente introdução. $\mathrm{Na}$ segunda parte faz-se uma revisão de trabalhos teóricos e empíricos sobre a relação entre produtividade e importações. Em seguida, na terceira parte, apresentam-se os dados e a metodologia. A quarta parte contém a análise dos resultados. Por fim, na quinta parte, alinham-se as principais conclusões sobre o trabalho.

${ }^{7}$ Ver Bezerra e Lima (2007) e Bonelli e Fonseca (2008). 


\section{IMPORTAÇÕES E PRODUTIVIDADE: REVISÃO DA LITERATURA}

Para Afonso (2001), a "Moderna Teoria do Crescimento Econômico" mudou a forma de se pensar a respeito dos impactos do comércio internacional sobre a produtividade e o crescimento econômico. De acordo com esse autor, o modelo de Solow ${ }^{8}$, que melhor representa essa teoria, prevê que o crescimento depende de fatores exógenos, a exemplo da poupança e do progresso tecnológico, este último influenciando a produtividade dos fatores. Dessa forma, o comércio internacional, que difunde tecnologias, passa a exercer efeitos pelo lado da oferta da economia, influenciando o nível e a taxa de crescimento da produtividade.

Assim também pensa Akamatsu (1962) apud Gonçalves et al. (1998), em sua teoria dos gansos voadores, segundo a qual uma economia ao importar de outra mais avançada absorve a tecnologia, o que lhe permite dinamizar a sua própria produção, ou, mesmo, a produzir o que antes importava. Bezerra e Lima (2005) destacam a evolução do pensamento neoclássico superando a necessidade de fatores ociosos, avançando para a realocação de fatores, e, então, para modelos dinâmicos nos quais há mudanças na função de produção. Essas mudanças podem ser causadas por insumos, fatores e/ou produtos importados.

De acordo com esses autores, dois aspectos são apontados pela literatura com o objetivo de relacionar o comércio internacional à produtividade, sendo eles: (i) o esforço empresarial apresenta maiores retornos em presença de concorrência externa; e, (ii) a ampliação do mercado, quando o país se insere no comércio externo, proporciona retornos crescentes.

\subsection{MODELOS BASEADOS NA TEORIA DO CRESCIMENTO ENDÓGENO}

Segundo Afonso (2001), a nova teoria do crescimento endógeno, utilizando-se de modelos de equilíbrio geral, destaca o papel das mudanças tecnológicas. Romer (1990) afirma que o estoque de capital humano determina a taxa de crescimento de uma economia, e que o comércio internacional contribui para que essa taxa alcance níveis mais elevados. Segundo o autor, o avanço tecnológico promove a acumulação de capital e, consequentemente, o crescimento. Ele conclui, ainda, que, quanto maior o mercado, maior é o incentivo à pesquisa, com as implicações naturais sobre o crescimento econômico?.

\footnotetext{
${ }^{8}$ Ver Solow (1957) apud Afonso (2001).

${ }^{9}$ Ver Romer (1990) para maiores detalhes do modelo.
} 
Grosman e Helpman (1990) apresentam um modelo de dois países que se dedicam a três atividades: à produção de um bem final, à produção de diferentes produtos intermediários e à pesquisa e desenvolvimento (P\&D). De acordo com o modelo, a taxa de crescimento em longo prazo se relaciona ao comércio através da difusão da tecnologia e do conhecimento ${ }^{10}$.

Grossman e Helpman (1991) argumentam sobre quatro canais em que o comércio causa o crescimento: incentivo aos empresários locais para realização de inovações, aumento do tamanho do mercado, promoção de uma melhor alocação dos recursos e, o mais importante, facilidade na aquisição de conhecimento. Para eles, o acesso a novos e melhores insumos e tecnologias promove o crescimento da produtividade ${ }^{11}$.

Rivera-Batiz e Romer (1991), a partir de um modelo no qual existem três efeitos das barreiras ao comércio externo ${ }^{12}$, concluem que na ausência de livre comércio há menos incentivo à pesquisa e ao desenvolvimento, menor possibilidade de transmissão de novos conhecimentos, e os países não alocam os recursos na produção de bens para os quais possuem vantagens comparativas. Sendo assim, as barreiras ao comércio tendem a reduzir o crescimento ${ }^{13}$.

Para Young (1991), os efeitos do comércio internacional diferem para nações desenvolvidas e em desenvolvimento, pois o processo de aprendizado produz efeitos de transbordamento entre os setores, implicando em que uma situação de comércio livre, os países desenvolvidos cresceriam mais rapidamente. $\mathrm{O}$ autor formula, por esse meio, um modelo de crescimento endógeno capaz de explicar a falta de convergência das taxas de crescimento entre as nações.

Kim e Kim (2000) associam o comércio internacional à acumulação de capital humano. O comércio é fundamental para que a mão de obra possa se transferir para o setor de maior produtividade em um dado período. A especialização implica que a taxa de crescimento dependerá do progresso técnico da indústria de maior produtividade. Enquanto isso, em uma economia fechada e de baixo nível de capital humano a expansão será dada pela média do crescimento de todas as indústrias. Assim, quanto maior for o capital humano de uma economia e maior for o seu coeficiente de abertura, mais elevada será sua taxa de crescimento.

Halpern et al. (2005) desenvolvem um modelo com firmas heterogêneas que decidem se devem importar, ou não, insumos intermediários. As importações afetam a produtividade das firmas por ampliarem tanto a variedade quanto a qualidade

\footnotetext{
${ }^{10}$ Ver Grossman e Helpman (1990) para maiores detalhes do modelo.

${ }^{11}$ Ver Grossman e Helpman (1991) para maiores detalhes do modelo.

${ }^{12}$ Os autores se basearam na teoria do consumidor, separando os efeitos das barreiras comerciais em: efeito de integração, de redundância e de alocação.

${ }^{13}$ Segundo os autores, em alguns casos as políticas comerciais podem contribuir para o crescimento econômico, principalmente, nas relações entre países em diferentes níveis de desenvolvimento.
} 
dos insumos. No modelo, a produtividade total dos fatores de uma firma depende da participação dos insumos importados. O teste empírico realizado pelos autores mostrou que as importações de insumos explicam 30\% do crescimento da produtividade total dos fatores na Hungria.

Kasahara e Lapham (2008), também através de um modelo com firmas heterogêneas, que, nesse caso, escolhem se devem exportar seus produtos e se devem importar insumos intermediários, mostram que a política de importações influencia a produtividade, a realocação de recursos e as exportações. Experimentos contrafactuais, realizados pelos autores, sugerem que é possível obter ganhos significativos de produtividade com a redução das barreiras comerciais.

Cavallo e Landry (2010), preocupados em compreender porque a importação de capital se tornou uma fonte importante de crescimento nos Estados Unidos, desenvolveram um modelo neoclássico no qual inserem o comércio internacional de bens de capital, e condicionam a alocação de recursos aos preços relativos desses bens, estrangeiros e domésticos. De acordo com essa formulação, o Estados Unidos teria apresentado um crescimento $20 \%$ menor desde 1967, caso não tivessem importado bens de capital.

\subsection{TRABALHOS EMPÍRICOS SOBRE A RELAÇÃO ENTRE IMPORTAÇÕES E PRODUTIVIDADE}

Lin e Wong (1997) estudaram os determinantes do crescimento de Hong Kong utilizando dados anuais de 1971 a 1990 e encontraram relação positiva entre o crescimento e as seguintes causas: acumulação de capital físico, redução do trabalho não qualificado, educação e os efeitos de transbordamento da tecnologia, em decorrência da importação de bens de capital.

Ainda no leste asiático, Lawrence e Weinstein (1999) procuraram evidências da ligação entre comércio e produtividade, em países da região. Para tanto, foram realizadas regressões com séries temporais, nas quais a variável dependente era o crescimento da Produtividade Total dos Fatores (PTF) a nível setorial. Um importante resultado foi que a abertura colaborou para o aumento na produtividade desses países, via efeitos da competição promovida pelas importações, destacando-se, nesse sentido, o Japão. Segundo os autores, "a concorrência de produtos importados estimula a inovação" (Lawrence e Weinstein, 1999, p. 24).

Choudhri e Hakura (2000), utilizando modelo desenvolvido por Krugman (1985), e dados de 33 países em desenvolvimento, encontram evidências de que o comércio internacional contribui para reduzir a desigualdade tecnológica entre setores industriais de diferentes países. Os autores concluem que os setores de baixo crescimento pouco se beneficiam do comércio. Nos setores de crescimento mediano, o aumento da 
concorrência devido às importações exerce um efeito expressivo. No caso dos setores de elevado crescimento, são as exportações que tendem a aumentar a produtividade.

Gosh et al. (2002) estimaram um modelo de vetores autorregressivos para analisar a relação entre diferença salarial, comércio internacional - exportações e importações - e crescimento da produtividade na economia americana, com dados anuais de 1967 a 1998. A produtividade do trabalho mostrou-se positivamente relacionada com as variáveis do comércio e com diferenças salariais em curto prazo.

Bernard et al. (2003), usando dados de firmas dos Estados Unidos e metodologia de equilíbrio geral, encontram evidências de que a diminuição das barreiras ao comércio tende a expulsar firmas de baixa produtividade e incentivar as firmas de alta produtividade a exportarem seus produtos.

Cameron et al. (2003) utilizaram um painel de dados de 14 setores da indústria do Reino Unido para estudar a importância da transferência de tecnologia e de investimentos em P\&D para o crescimento da produtividade total dos fatores. Os resultados indicam que o comércio internacional contribui para aumentos de produtividade através da transferência de tecnologia.

Hung et al. (2004) mostram que um declínio no preço das importações promove um crescimento da PTF com defasagem de 1 ou 2 anos; um aumento do volume das importações provoca um aumento na produtividade do trabalho; a correlação entre o nível das exportações e a produtividade desaparece em um ano. Esse último resultado é uma forte evidência de que as exportações não têm impacto persistente sobre a produtividade.

Alguns estudos do gênero foram realizados para o caso brasileiro e sua indústria de transformação. Dentre eles, Sabóia e Carvalho (1997) estudaram a abertura comercial como uma das explicações para o crescimento da produtividade industrial, observado a partir do início da década de 1990. Utilizando a análise das correlações entre as séries, as medidas de exportação apresentaram coeficientes de correlação positivos e significantes. Para os autores, a abertura da economia obrigou as empresas a racionalizar o processo produtivo para enfrentar um ambiente mais competitivo, e aqueles setores em que mais cresceu a produtividade tenderam a apresentar resultados mais favoráveis, em termos de exportações. Em contrapartida, nenhuma das medidas de importação mostrou-se significativamente correlacionada com a produtividade, o que, segundo os autores, pode resultar de forças contraditórias, a saber: por um lado, as importações cresciam em setores de muito baixa produtividade; por outro, os setores de alta produtividade estavam tentando se defender da competição dos produtos importados. Esses efeitos estariam anulando a correlação entre a produtividade e as importações. 
Lisboa et al. (2000) estudaram os canais de impacto da abertura comercial sobre as tecnologias adotadas. Para um conjunto de dados referentes à cerca de 1.700 empresas, entre 1988 a 1998, os autores verificaram que a redução nas tarifas de importação de insumos foi a principal responsável pelo crescimento da produtividade das firmas brasileiras no período analisado.

Ferreira e Rossi Jr. (2003), a partir de um painel de dados referentes a 16 setores da indústria de transformação, entre 1985 a 1997, procuraram relação entre crescimento da produtividade e abertura comercial. Foram estimados três modelos. Para os dois primeiros, as variáveis dependentes eram duas medidas para a produtividade do trabalho - produção por trabalhador empregado e produção por hora de trabalho contratada - e para o terceiro, era a produtividade total dos fatores. As variáveis explicativas eram as taxas de proteção, nominais e efetivas, além de dummies por setor, dummies de tempo, e variáveis de controle, como a inflação. Os resultados indicaram uma relação negativa entre a proteção tarifária e o crescimento da produtividade, sendo robustos com relação a mudanças na metodologia, nas variáveis e no tempo.

Hay (2001), considerando dados de 318 firmas de manufaturas entre 1986 e 1994, encontram evidências de que a abertura comercial promoveu um aumento de produtividade acompanhado de redução na participação de mercado e nos lucros. $\mathrm{O}$ autor sugere que o choque da liberalização comercial nos lucros estimulou as firmas a buscarem formas mais eficientes de produção.

Ferreira e Guillén (2004), com dados de 16 setores de 1985 a 1997, observaram que a abertura comercial promoveu ganhos em produtividade na indústria, possivelmente através do acesso a insumos importados do uso de novas tecnologias, e não pelo aumento da concorrência.

Por sua vez, Silva (2004) comprovou que o efeito da abertura comercial sobre a produtividade total dos fatores da indústria de transformação é positivo e significante de modo agregado. Porém, essa relação não foi observada em alguns dos setores quando considerados isoladamente. A autora utilizou dados em painel de 47 setores da indústria brasileira entre 1989 e 1999.

Muendler (2004), estudando os efeitos da redução da proteção tarifária no Brasil, a partir de 1990, sobre a produtividade das firmas de médio e grande porte, com dados da PIA-IBGE referentes à cerca de 9.500 firmas entre 1986 e 1998, observa que o canal da competitividade é uma importante fonte de mudanças na produtividade, que a saída de firmas ineficientes aumenta a produtividade apenas gradualmente e que o efeito do uso de insumos e equipamentos estrangeiros é praticamente nulo na média, apesar de ser observado para alguns setores.

Bezerra e Lima (2005) concluíram que existe uma relação entre o comércio internacional e a produtividade na região Nordeste do Brasil. Adicionalmente, os 
resultados obtidos sugerem que os efeitos na produtividade são um fenômeno de oferta, como explica a teoria do crescimento endógeno. Nesse trabalho foram utilizados dados anuais da região Nordeste, e dados mensais da indústria da mesma região, a produtividade foi medida como o produto por trabalhador, e a análise foi realizada sob duas diferentes técnicas estatísticas: regressões lineares e vetores autorregressivos (VAR).

Bonelli e Pinheiro (2008) investigaram o impacto da abertura comercial sobre o crescimento da produtividade, levando em conta o crescimento da inserção de produtos importados. Os autores estimaram regressões do crescimento médio da produtividade do trabalho, para a economia como um todo, entre 1990 e 2000, contra as seguintes variáveis: o nível absoluto e as taxas de variação da proteção nominal e da proteção efetiva, e o coeficiente de inserção das importações. Em nenhum dos casos foi possível estabelecer uma relação estatística significante. Contudo, observando o desempenho individual dos setores, eles concluíram que em alguns deles o crescimento foi uma resposta à abertura comercial e ao aumento da competição, principalmente entre aqueles em que o coeficiente de penetração das importações já superava a média antes da abertura, a exemplo dos setores automobilístico, de equipamentos elétricos, $\mathrm{e}$ de produtos de borracha. Em outros, como a agricultura e o setor têxtil, o benefício da abertura se deu sob a forma de melhores insumos, máquinas e equipamentos.

\section{OS DADOS E A ESTRATÉGIA EMPÍRICA}

Uma parcela considerável dos trabalhos empíricos sobre a relação entre a produtividade e a abertura comercial tem utilizado técnicas do tipo cross-section de países.

Segundo Giles e Williams (2000), vários autores argumentaram que esse tipo de análise apresenta problemas, tais como: indefinição de direção de causalidade devido à endogeneidade das variáveis; o fato de não considerarem as diferentes características econômicas e institucionais dos países, o uso de taxas médias de crescimento de conjuntos de países, produzindo instabilidade dos parâmetros estimados etc. Contudo, segundo ainda aqueles autores, o pior problema se deve ao fato de que esse tipo de análise não consegue distinguir facilmente quando existe uma relação causal entre duas variáveis, e quando elas estão simplesmente correlacionadas, neste caso refletindo os efeitos comuns originados em uma terceira variável. Por esses motivos, a análise por meio de séries temporais é mais recomendada se o objetivo é verificar a relação entre duas variáveis.

Dentre as principais técnicas aplicáveis nesse tipo de análise, destacam-se o teste de causalidade de Granger e os modelos de vetores autorregressivos, os quais serão utilizados aqui e, cujos detalhes, são apresentados a seguir. As estimativas e testes foram realizados com o uso do software Regression Analysis of Time Series (RATS), versão 7.0. 


\subsection{OS DADOS}

Os dados são de periodicidade trimestral, com início em 1992:1, término em 2008:2, e compreendem as seguintes amostras: índice de produção física industrial, obtido no Instituto Brasileiro de Geografia e Estatística (IBGE); índice das horas trabalhadas na indústria de transformação, obtido na Confederação Nacional da Indústria (CNI); valores (US\$ FOB) das importações do Brasil obtidos do MDIC; índice de preços ao atacado (IPA) dos Estados Unidos, obtido do Bureau of Labor Statistics (BLS) ${ }^{14}$.

Os valores das importações medidos a preços correntes em dólares americanos foram deflacionados com o índice de preços ao atacado (IPA) dos Estados Unidos.

A estimativa da produtividade do trabalho foi obtida através da divisão do índice de produção industrial da PIM- $\mathrm{PF}^{15}$ pelo índice do número de horas trabalhadas na produção calculado pela CNI, tal como divulgado através do Banco Central do Brasil (2009).

Segundo Netto e Curado (2005), a produtividade por hora é mais precisa que a produtividade por trabalhador, pois incorpora a jornada de trabalho. Esses autores argumentam que a produção por trabalhador pode aumentar simplesmente por aquele estar trabalhando mais tempo, sem significar uma produção maior por hora.

Com relação aos dados da produção industrial, há uma mudança metodológica em 2002, porém, segundo o IBGE, todos os dados, desde 1991, foram reformulados ${ }^{16}$ para manter a compatibilidade. Há um questionamento quanto à representatividade da PIM-PF. Nesse sentido, vale lembrar que, segundo o IBGE, a cobertura de produtos da amostra da PIM-PF em termos do valor da transformação industrial (VTI), corresponde a apenas $61,8 \%$ para o território nacional ${ }^{17}$.

\footnotetext{
${ }^{14}$ Informações a respeito da metodologia aplicada pelas instituições responsáveis pela elaboração das informações utilizadas nessa pesquisa podem ser encontradas nos seguintes endereços eletrônicos:

http://www.ibge.gov.br/home/estatistica/indicadores/industria/pimpfbr/notas_metodologicas.shtm; http://www.cni.org.br/termometro/horastrabalhadas.htm;

http://www.mdic.gov.br//sitio/interna/interna.php?area=5\&menu=605; http://www.bls.gov/ppi/methodology.htm.

${ }^{15}$ Não existe uma série do valor adicionado da indústria de transformação, por isso foi utilizado um índice de quantidade produzida como proxy.

${ }^{16}$ Ver http://www.ibge.gov.br/home/estatistica/indicadores/industria/pimpfbr/default.shtm.

${ }^{17}$ Ver IBGE (2004).
} 


\subsection{ESTRATÉGIA EMPÍRICA}

\subsubsection{TESTE DE CAUSALIDADE DE GRANGER}

Para determinar a existência de causalidade entre importações e produtividade, foi realizado o teste de causalidade de Granger (1969). Neste item explicam-se o significado do teste e o critério estatístico de avaliação adotado. Os resultados encontram-se na seção 4.1.

Para testar se uma variável $X$ Granger causa a variável $Y$, estima-se uma regressão do tipo:

$$
Y_{t}=\sum_{i=1}^{m} \alpha_{i} X_{t-i}+\beta_{i} Y_{t-i}+\mu_{t}
$$

Então, testa-se a hipótese nula de que:

$$
H_{0}: \alpha_{1}=\ldots=\alpha_{n}=0
$$

A estatística de teste é dada por:

$$
\frac{\left(R S S_{R}-R S S_{U}\right) / m}{R S S_{U} /(n-k)}
$$

em que $R S S_{R}$ é a soma dos quadrados dos resíduos do modelo restrito (estimado sem os coeficientes $\alpha_{i}$ ), RSS $S_{U}$ é a soma dos quadrados dos resíduos do modelo irrestrito, $m$ é o número de termos defasados associados à variável $X, n$ é o número de observações e $k$ é o número de parâmetros estimados no modelo irrestrito. Essa estatística segue distribuição $F_{m, n-k}$. A hipótese nula foi rejeitada sempre que o valor calculado da estatística $F$ foi maior, em termos absolutos, que o valor crítico a um nível de significância de $5 \%$.

Para determinar o número de defasagens das variáveis foram usados os critérios de seleção de Akaike (AIC), e Schwarz (SBC).

\subsubsection{OS MODELOS DE VETORES AUTORREGRESSIVOS}

Um dos principais avanços da análise de séries temporais são os modelos de vetores autorregressivos (VAR). O VAR consiste em um sistema de equações, em que cada uma das variáveis que compõem o sistema é função dos valores das demais variáveis no presente, dos seus valores e dos valores das demais variáveis defasadas no tempo, mais o termo de erro. As equações de um modelo VAR podem conter também tendências determinísticas e variáveis exógenas. A partir de algumas operações matemáticas o 
modelo VAR pode ser transformado de modo que, nas equações, os valores do presente deixam de constar como variáveis explicativas ${ }^{18}$. Esta é a forma conhecida como VAR reduzido (Enders, 2004).

A partir de um modelo de vetores autorregressivos, são estimadas a função impulso-resposta e a decomposição da variância. Utilizando a função de impulso-resposta, é possível perceber como uma variação ocorrida em uma das variáveis do sistema repercute nas demais em um determinado horizonte de tempo ${ }^{19}$. A decomposição da variância, por sua vez, revela a proporção da variância do erro de previsão para uma das variáveis que se deve a ela mesma, e às demais (Enders, 2004).

Ainda com relação ao modelo VAR, se mais de uma das séries que se pretende incorporar ao sistema não forem estacionárias e de mesma ordem de integração, então é provável que haja uma relação de cointegração entre elas, ou seja, apresentam uma tendência em longo prazo comum (Enders, 2004). Existindo essa relação, que é confirmada a partir de um teste de cointegração, o modelo VAR não é o método mais indicado para a análise das séries, pois seus resultados seriam estatisticamente inconsistentes. Nesses casos, deve-se usar o método dos vetores com correção de erro (VEC) (Johnston e DiNardo, 2001).

Um modelo VEC é semelhante a um VAR, porém em todas as equações do primeiro está contido um vetor de correção de erro, que, como sugere o nome, tem como objetivo corrigir as relações de cointegração (Johnston e DiNardo, 2001). Segundo Enders (2004), um modelo VEC com apenas um vetor de cointegração pode ser representado da seguinte forma:

$$
\Delta x_{t}=\sum_{i=1}^{k} \beta \Delta x_{t=i}+\beta a^{\prime} x_{t-1}+\varepsilon_{t}
$$

Em que $x$ é o vetor das variáveis, $a^{\prime} x_{t-1}$ é o vetor de cointegração e $k$ é o número de defasagens.

\subsubsection{TESTES DE HIPÓTESE SOBRE AS CARACTERÍSTICAS DAS SÉRIES}

O teste de Dickey-Fuller aumentado (ADF) é uma metodologia utilizada para determinar se as séries são estacionárias. Assumindo que os erros são independentes e a

\footnotetext{
${ }^{18}$ Segundo Enders (2004), essa transformação é necessária, pois não é possível estimar o modelo em sua forma primitiva. A razão é que os valores presentes das variáveis do sistema são correlacionados com os termos de erro das equações. Assim, para encontrar o VAR primitivo, é preciso estimar a forma reduzida. ${ }^{19}$ Para identificar o efeito de um choque de uma das variáveis do sistema em outras no tempo presente, é preciso recuperar a formulação primitiva do VAR. No entanto, como o número de coeficientes do VAR reduzido é inferior, não é possível determinar todos os coeficientes do VAR primitivo, alguns deles devem ser restringidos a zero. Neste estudo, utiliza-se Decomposição de Choleski, assim, na matriz dos coeficientes presentes, restringe-se a zero todo coeficiente abaixo da diagonal principal (Enders, 2004).
} 
variância é constante, testa-se a hipótese de que o parâmetro g é igual à zero nas três formulações a seguir:

$$
\begin{gathered}
\Delta y_{t}=\gamma y_{t-1}+\sum_{i=1}^{p} \beta_{i} \Delta y_{t-i}+\varepsilon_{t} \\
\Delta y_{t}=a_{0}+\gamma y_{t-1}+\sum_{i=1}^{p} \beta_{i} \Delta y_{t-i}+\varepsilon_{t} \\
\Delta y_{t}=a_{0}+a_{1} t+\gamma y_{t-1}+\sum_{i=1}^{p} \beta_{i} \Delta y_{t-i}+\varepsilon_{t}
\end{gathered}
$$

a distribuição do teste é $\tau$ (tau) e a série será estacionária se a hipótese nula puder ser rejeitada, o que ocorre no caso em que o valor calculado seja menor do que o valor crítico a um nível de significância de 5\%.

Para determinar a ordem de defasagens $(p)$ das equações (1), (2) e (3), foi utilizado o critério dos multiplicadores de Lagrange.

Uma série estacionária que apresenta quebra estrutural enviesa o resultado do teste ADF, que tende a acusar a presença de uma raiz unitária. Para evitar esse problema, utiliza-se a metodologia desenvolvida por Phillips e Perron (1989), o teste de Phillips-Perron (doravante PP). Esse procedimento envolve três hipóteses:

$$
\begin{aligned}
& H_{0}: \mu_{1}=0 \\
& H_{1}: \mu_{1} \neq 0
\end{aligned} ; \begin{aligned}
& H_{0}: \mu_{2}=0 \\
& H_{1}: \mu_{2} \neq 0
\end{aligned} \text { e } \begin{aligned}
& H_{0}: a_{1}=1 \\
& H_{1}: a_{1}<1
\end{aligned}
$$

para a equação:

$$
\Delta y_{t}=a_{0}+\mu_{1} D_{l}+\mu_{2} D_{p}+a_{1} y_{t-1}+a_{2} t+\sum_{i=1}^{k} \beta_{i} \Delta y_{t-i}+\varepsilon_{t}
$$

os testes seguem a distribuição $t$, e as hipóteses nulas indicam que não há quebra estrutural (nível ou pulso) e que a série possui raiz unitária. As hipóteses nulas são rejeitadas sempre que o valor calculado seja menor do que o valor crítico a um nível de significância de $5 \%$.

É possível que uma série se torne estacionária apenas se for diferenciada de forma sazonal, nesse caso diz-se que a série possui raiz unitária sazonal. Para identificar essa possibilidade em séries de dados trimestrais utiliza-se o procedimento desenvolvido por Hylleberg et al. (1990), o teste HEGY. A partir da equação, a seguir:

$$
\left(1-L^{4}\right) y_{t}=\gamma_{1} y_{1 t-1}-\gamma_{2} y_{2 t-1}+\gamma_{5} y_{3 t-1}-\gamma_{6} y_{3 t-2}+\varepsilon_{t}
$$


em que:

$$
\begin{gathered}
y_{1 t-1}=\left(1+L+L^{2}+L^{3}\right) y_{t-1} \\
y_{2 t-1}=\left(1-L+L^{2}-L^{3}\right) y_{t-1} \\
y_{3 t-1}=\left(1-L^{2}\right) y_{t-1}
\end{gathered}
$$

se a hipótese de $\gamma_{1}=0$ não é rejeitada, seguindo distribuição $t$, então podemos concluir que há uma raiz unitária não sazonal. Caso a hipótese nula para $\gamma_{2}=0$ não seja rejeitada, indica a presença de uma raiz unitária com frequência semianual. E, a hipótese nula para $\gamma_{5}=\gamma_{6}=0$, com base na distribuição $F$, sugere a presença de uma raiz unitária sazonal. As hipóteses não são mutuamente excludentes, ou seja, a presença de uma raiz unitária sazonal não exclui a possibilidade da presença de outra raiz unitária não sazonal (Enders, 2004).

As hipóteses nulas são rejeitadas sempre que o valor calculado seja menor do que o valor crítico a um nível de significância de 5\%.

\subsection{ANÁLISE DE COINTEGRAÇÃO}

Como foi dito anteriormente, caso duas ou mais variáveis sejam integradas de mesma ordem, em outras palavras, precisem do mesmo número de diferenciações para que se tornem estacionárias, então pode existir uma combinação linear entre elas que seja estacionária, e quando isso ocorre se diz que as variáveis são cointegradas ${ }^{20}$.

Para testar a possibilidade de cointegração entre variáveis de mesma ordem de integração foi utilizado o teste de máxima verossimilhança desenvolvido por Johansen (1988). O teste pode ser visto como uma generalização do teste Dickey-Fuller para o ambiente multivariado (Enders, 2004). Seja a equação:

$$
x_{t}=A_{1} x_{t-1}+\varepsilon_{t}
$$

então:

$$
\Delta x_{t}=\left(A_{1}-I\right) x_{t-1}+\varepsilon_{t}=\pi x_{t-1}+\varepsilon_{t}
$$

em que $x t$ e $\varepsilon_{t}$ são vetores $n_{x}, 1 I$ é uma matriz-identidade $n_{x} n, A 1$ e $\pi$ são matrizes $n_{x} n$. De modo análogo ao teste Dickey-Fuller, se o posto de $\pi$ for igual à zero, então

\footnotetext{
${ }^{20}$ Segundo Enders (2004), considera-se equilíbrio em longo prazo entre as $n$ variáveis do vetor $X$ quando:

$\beta_{1} x_{1 t}+\beta_{2} x_{2 t}+\ldots+\beta_{n} x_{n t}=0$

$\mathrm{O}$ desvio do equilíbrio em longo prazo, $e_{t}$, é dado por:

$e_{t}=\beta X_{t}$
}

Se o equilíbrio existe, o processo de $e_{t}$ deve ser estacionário, e o vetor $\beta$ é chamado de vetor de cointegração. 
toda a sequência em $x_{1}$ possui raiz unitária, as variáveis não são cointegradas. Mas, se o posto de $\pi$ for $n \geq 1$, e as raízes características (autovalores) dessa matriz forem maiores que a unidade, então os resíduos da combinação linear são estacionários, e as variáveis são cointegradas.

O teste consiste em verificar a significância das raízes características da matriz $\pi$, o número de vetores de cointegração é igual ao número de autovalores significantes. Para tanto, calcula-se a estatística de traço:

$$
\lambda_{\text {traço }}(r)=-T \sum_{i=r+1}^{n} \ln \left(1-\hat{\lambda}_{i}\right)
$$

em que $\hat{\lambda}_{i}$ são os valores estimados dos autovalores da matriz $\pi$, e $T$ é o número de observações utilizáveis. A partir da estatística de traço, testa-se a hipótese nula de que o número de vetores de cointegração independentes é menor ou igual a $r$. Os valores críticos para o teste foram obtidos por Johansen (1988) a partir do procedimento Monte Carlo (Enders, 2004).

Note-se que os testes de cointegração dependem do número de defasagens do modelo, por isso, são realizados considerando modelos com diferentes defasagens. Neste trabalho, apresenta-se apenas o modelo que não apresentou resíduos autocorrelacionados, que foi o de quatro defasagens, conforme demonstraram os testes de Ljung-Box e dos multiplicadores de Lagrange ${ }^{21}$.

Uma vez constatado que duas ou mais variáveis são cointegradas, é importante identificar se alguma delas pode ser considerada fracamente exógena em relação ao vetor de cointegração. Isso ocorre se o parâmetro que representa sua velocidade de ajustamento a desequilíbrios temporários na relação em longo prazo não for estatisticamente diferente de zero. Para testar essa hipótese, utiliza-se a estatística de razão de máxima verossimilhança.

Também é válido verificar se a relação de cointegração é estável ao longo de toda a amostra. Para tanto, será utilizado o procedimento recursivo do $\beta$ conhecido. A hipótese nula do teste afirma que valores estimados para $\beta$ com a amostra completa, denominados $\beta_{0}$, estão contidos no espaço gerado por $\hat{\beta}^{(n)}-$ a estimação do vetor $\beta$ considerando a n-ésima amostra. $O$ procedimento é recursivo, pois os parâmetros são reestimados a partir de amostras menores, que se iniciam no menor período necessário para que possam ser estimados, sendo acrescentados em seguida os demais períodos progressivamente. Esse processo é realizado de duas formas, reestimando todos

${ }^{21}$ Ver Hansen e Juselius (1995) para maiores detalhes a respeito dos testes estatísticos para autocorrelação dos resíduos, exogeneidade fraca e $\beta$ conhecido. 
os parâmetros do modelo ( $X$-form), e reestimando apenas os parâmetros do vetor de cointegração (R1-form).

\section{ANÁLISE DOS RESULTADOS}

Como forma de analisar a influência do volume de importações sobre a produtividade, realizou-se o teste de causalidade de Granger e a técnica dos vetores autorregressivos. Os modelos foram estimados relacionando a produtividade do trabalho (PRODHT), medida como a relação entre a produção física industrial e as horas de trabalho pagas na produção, com o valor real das importações $(M)$.

Primeiramente, porém, buscou-se identificar a ordem de integração das séries. Os resultados dos testes ADF, PP e HEGY, indicaram que as séries são todas integradas de primeira ordem e não possuem quebras estruturais. Ademais, identificou-se que a série PRODHT apresenta raiz unitária sazonal. Os resultados desses testes estão no apêndice Tabelas 7, 8, 9 e 10). Em todos os casos, foi considerado o nível de significância de 5\% para a rejeição da hipótese nula.

\subsection{TESTE DE CAUSALIDADE DE GRANGER}

O primeiro procedimento realizado na tentativa de analisar a relação entre produtividade e importações foi o teste de causalidade de Granger. Nesse sentido, primeiramente, buscou-se determinar a ordem de defasagens do teste, para tanto foram considerados os critérios de seleção de Akaike (AIC) e Schwartz (SBC). Os resultados, organizados na Tabela 1 abaixo, indicam a seleção do modelo com quatro defasagens.

O teste de causalidade de Granger foi, então, realizado considerando quatro defasagens e 5\% de nível de significância. Os resultados, apresentados na Tabela 2, indicam a bicausalidade entre PRODHT e M. Dessa forma, justifica-se a estimação de um modelo de vetores autorregressivos como forma de analisar a relação entre as variáveis.

Tabela 1 - Critérios de seleção do número de defasagens

\begin{tabular}{l|c|c}
\hline Defasagens & AIC & SBC \\
\hline 0 & $1.611,58$ & $1.615,73$ \\
\hline 1 & $1.352,26$ & $1.364,30$ \\
\hline 2 & $1.359,23$ & $1.378,55$ \\
\hline 3 & $1.318,03$ & $1.343,95$ \\
\hline 4 & $1.299,89$ & $1.331,66$ \\
\hline
\end{tabular}

Fonte: Elaboração própria. 
Tabela 2 - Teste de causalidade de Granger (quatro defasagens)

\begin{tabular}{|c|c|c|c|c|}
\hline & \multicolumn{4}{|c|}{ Variável Dependente } \\
\hline & \multicolumn{2}{|c|}{ PRODHT } & \multicolumn{2}{|c|}{$\mathbf{M}$} \\
\hline & F-Calculado & P-valor & F-Calculado & P-valor \\
\hline PRODHT & 204,3784 & 0,000000 & 2,5687 & 0,0484414 \\
\hline M & 3,3854 & 0,015384 & 63,2372 & 0,000000 \\
\hline
\end{tabular}

Fonte: Elaboração própria.

\subsection{MODELO PRODHT E M}

Como PRODHT e M são não estacionárias de mesma ordem de integração, é possível existir entre elas uma tendência comum em longo prazo. Em outras palavras, há possibilidade de cointegração. Assim, antes da estimação do modelo, testou-se essa hipótese através do procedimento de Johansen. Se essa hipótese não for rejeitada, então o modelo VAR deve incorporar o vetor de cointegração estimado - caracterizando um modelo de correção de erro (VEC).

Antes de realizar o teste de Johansen, é preciso determinar a ordem de defasagem a ser utilizada. Conforme pode ser obervado na Tabela 3, o modelo com quatro defasagens é adequado, pois os testes de resíduos autocorrelacionados pelos critérios de Ljung-Box e Multiplicadores de Lagrange rejeitam essa hipótese.

Tabela 3 - Testes de autocorrelação dos resíduos - modelo: PRODHT e M

\begin{tabular}{l|c|c|c}
\hline Teste & Graus de Liberdade da Distribuição $\chi^{\mathbf{2}}$ & Estatística & Significância \\
\hline Ljung-Box (15) & 46 & 43,926 & {$[0,560]$} \\
\hline $\operatorname{LM}(1)$ & 4 & 6,526 & {$[0,163]$} \\
\hline $\operatorname{LM}(2)$ & 4 & 5,055 & {$[0,282]$} \\
\hline
\end{tabular}

Fonte: Elaboração própria.

Os resultados do teste de cointegração indicam que existe uma tendência comum em longo prazo entre as séries, conforme pode ser visto na Tabela 4 . O procedimento foi realizado considerando um modelo com quatro defasagens das variáveis e dummies sazonais (uma vez que a série da variável PRODHT apresenta raiz unitária sazonal). Os resultados são significantes ao de $5 \%$. 
Tabela 4 - Teste de Johansen para cointegração - modelo: PRODHT e M

\begin{tabular}{l|c|c|c|c}
\hline Hipótese Nula & Autovalor & Estatística de Traço & Valor Crítico (5\% n.s.) & P-Valor \\
\hline$r \leq 0$ & 0,216 & 15,654 & 15,408 & 0,046 \\
\hline$r \leq 1$ & 0,009 & 0,544 & 3,841 & 0,461 \\
\hline
\end{tabular}

Fonte: Elaboração própria.

Em seguida, foi estimado o vetor de cointegração, apresentado na Tabela 5. O teste do Beta Conhecido indica que esse vetor de cointegração é estável ao longo de todo o período da amostra, de acordo com os métodos $\mathrm{R}(\mathrm{t})$ e $\mathrm{X}(\mathrm{t})$. Os resultados desse procedimento encontram-se no apêndice.

Tabela 5 - Vetor de cointegração - modelo: PRODHT e M

\begin{tabular}{l|c|c}
\hline Variável & PRODHT & M \\
\hline Coeficiente & 1 & $-0,011$ \\
\hline (t-student) & - & $(-2,725)$ \\
\hline
\end{tabular}

Fonte: Elaboração própria.

Considerando o vetor de cointegração apresentado acima, estimou-se o modelo de vetores autorregressivos corrigido (VEC). A decomposição da variância do modelo indica que $M$ chega a explicar mais de $45 \%$ da variância do erro de previsão de PRODHT, para previsão de 24 trimestres à frente. PRODHT, por sua vez, tem um menor poder de explicação da variância do erro de previsão de $M$, chegando a mais de 15\% para previsões de 24 trimestres à frente. Esses valores estão organizados na Tabela 6.

Os gráficos das funções de impulso-resposta mostram que as variáveis estão positivamente relacionadas com o longo prazo, conforme pode ser visto na Figura 1. Um choque positivo de um desvio-padrão na produtividade possui um impacto positivo sobre as importações desde o primeiro trimestre. As funções impulso-resposta estão compreendidas em intervalos de confiança de $95 \%$.

Tabela 6 - Decomposição da variância do erro de previsão - modelo: PRODHT e M

\begin{tabular}{l|c|c|c|c}
\hline \multicolumn{3}{|c|}{ PRODHT } & \multicolumn{2}{c}{ M } \\
\hline Período & PRODHT & M & PRODHT & M \\
\hline 1 & 100 & 0 & 0,376 & 99,624 \\
\hline 5 & 94,843 & 5,157 & 15,069 & 84,931 \\
\hline 10 & 92,827 & 7,173 & 15,865 & 84,135 \\
\hline 15 & 79,571 & 20,429 & 15,904 & 84,096 \\
\hline 20 & 64,273 & 35,727 & 15,805 & 84,195 \\
\hline 24 & 54,362 & 45,638 & 15,702 & 84,298 \\
\hline
\end{tabular}

Fonte: Elaboração própria. 
No caso de um choque nas importações, o efeito positivo somente aparece a partir do quinto trimestre. $\mathrm{O}$ impacto negativo das importações sobre a produtividade nos primeiros trimestres é compreensível, pois, um aumento das importações tende a diminuir a produção doméstica devido à concorrência, o que representa uma queda na relação produto por horas trabalhadas - medida utilizada para produtividade.

Figura 1 - Funções impulso-resposta

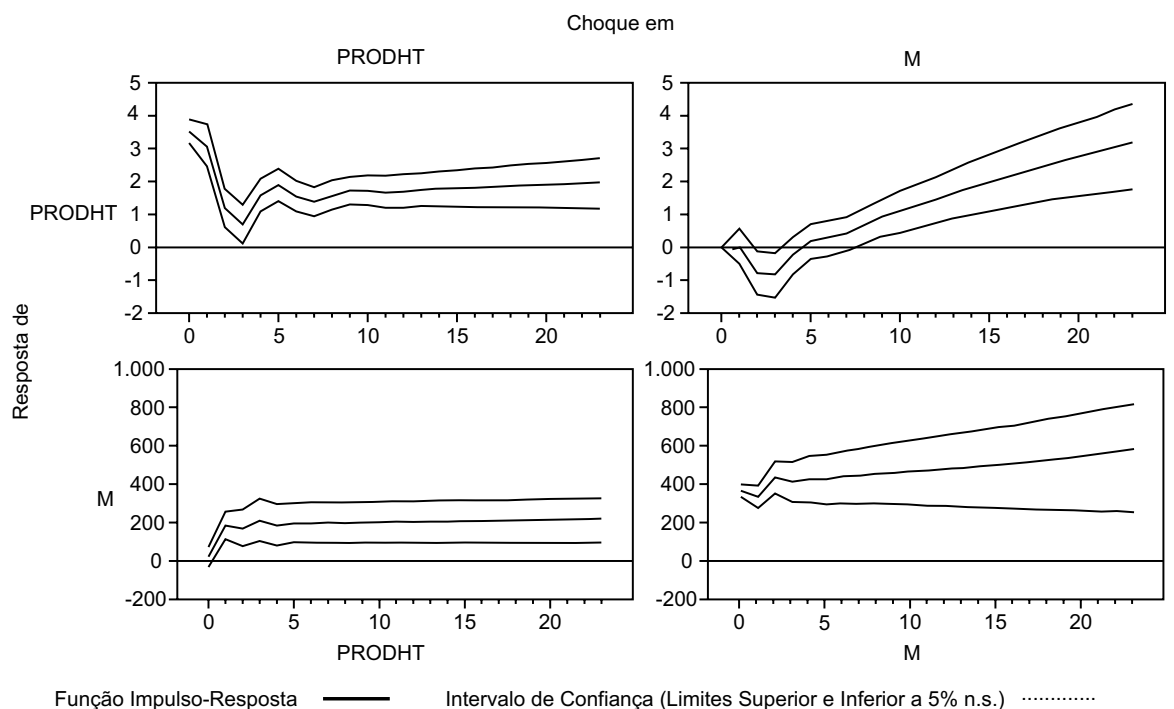

Fonte: Elaboração própria.

Os resultados encontrados estão coerentes com o que versa a teoria do crescimento endógeno. Segundo essa linha de pensamento, o comércio internacional contribui para ganhos de produtividade, pois permite o acesso a novas tecnologias e melhores insumos, além de aumentar a concorrência, incentivando a busca por técnicas mais eficientes de produção.

\section{CONCLUSÕES}

Este trabalho teve por objetivo examinar a relação entre o volume das importações (M) e a produtividade do trabalho na indústria de transformação do Brasil (PRODHT). Para identificar a existência dessa relação, foram utilizados o teste de causalidade de Granger e a técnica dos vetores autorregressivos com correção de erro. Os modelos foram estimados relacionando a produtividade do trabalho, medida como a relação entre a produção física industrial e as horas de trabalho pagas na produção, com o volume das importações. 
Os resultados do teste de causalidade de Granger indicaram a bicausalidade entre o volume das importações M e PRODHT, considerando quatro defasagens (número determinado pelos critérios de seleção AIC e SBC) e um nível de significância de 5\%. O teste F produziu p-valor de 0,015 para M e 0,048 para PRODHT, confirmando a existência de bicausalidade.

Os testes ADF, PP e HEGY indicaram que as duas séries são integradas de primeira ordem e não possuem quebras estruturais. Além disso, o teste HEGY indicou a presença de raiz unitária sazonal para a série da produtividade. Os valores críticos e as estatísticas desses testes encontram-se nas Tabelas 7 a 10 do apêndice, mostrando que todas são significantes a níveis de $5 \%$.

Uma vez que as séries da produtividade e do volume das importações são integradas de mesma ordem, a existência de apenas um vetor de cointegração entre $\mathrm{M}$ e PRODHT foi constatada a partir do teste de Johansen com um valor da estatística de traço igual a 15,654 .

Como forma de ampliar o entendimento a respeito da relação entre as duas variáveis, estimou-se um modelo de vetores autorregressivos com correção de erro, e foram realizados os exercícios de decomposição da variância do erro de previsão e de funções de impulso-resposta.

A decomposição da variância do erro de previsão indica que as importações explicam $45 \%$ da variância do erro de previsão da produtividade, enquanto esta explica apenas $15 \%$ da variância do erro de previsão das importações. Pela função impulso-resposta, um aumento das importações implica em redução da produtividade nos primeiros períodos, provavelmente porque aumenta a concorrência no mercado interno, diminuindo a participação no mercado e a produção das empresas locais.

A análise desenvolvida neste trabalho disponibiliza aos formuladores de políticas econômicas informações importantes. Em meio à controvérsia no que concerne aos efeitos das importações sobre a indústria brasileira, o estudo confirma evidencias, presentes tanto na literatura teórica quanto nos trabalhos empíricos, de que por essa via é possível promover-se o crescimento da produtividade industrial, o que gera incontestáveis benefícios para a competitividade e para o crescimento da economia. Talvez seja preciso de uma estratégia inteligente para se promover esses benefícios.

\section{REFERÊNCIAS}

AFONSO, O. The Impact of International Trade on Economic Growth. Investigação - Trabalhos em Curso, n. 106, maio, 2001.

ARBACHE, J.S.; CORSEUIL, C.H. Liberalização comercial e estruturas de emprego e salário. Texto para Discussão, n. 801, IPEA, Brasília, 2001.

AVERBUG, A. Abertura e Integração Comercial Brasileira na Década de 90. In: GIAMBIAGI, F; MOREIRA, M.M. (orgs.). A economia brasileira nos anos 90. Rio de Janeiro: BNDES, 1999. 
BERNARD, A.B.; EATON, J.; JENSEN, J.; KORTUM, S. Plants and Productivity in International Trade. The American Economic Review, v. 93, n. 4, p. 1.268-1.290, 2003.

BEZERRA, J.; LIMA R. Efeitos do Comércio Internacional sobre a Produtividade: A Evidência Empírica para o Nordeste Brasileiro Utilizando Vetores Autorregressivos (VAR). In: Encontro Regional de Economia, 10, Fortaleza, Anais, Fortaleza: Fórum BNB de Desenvolvimento, 2005.

- A liberalização do comércio internacional e a produtividade da indústria: a evidência empírica para o nordeste brasileiro utilizando vetores de correção de erro (VEC). In: Encontro Regional de Economia, 12, Fortaleza, Anais, Fortaleza: BNB, 2007.

BONELLI, R.; FONSECA, R. Ganhos de Produtividade e de Eficiência: Novos Resultados para a Economia Brasileira. Texto para Discussão, n. 557, IPEA, Brasília, 1998.

BONELLI, R.; PINHEIRO, A.C. Abertura e Crescimento Econômico no Brasil. In: GIAMBIAGI, F.; BARROS, O. (orgs.). Brasil globalizado. Rio de Janeiro: Elsevier, 2008.

BONELLI, R.; VEIGA, P.M.; BRITO, A.F. A Política Industrial e de Comércio Exterior no Brasil: rumos e indefinições. Texto para Discussão, n. 527, IPEA, Rio de Janeiro, 1997.

CAMERON, G.; PROUDMAN, J.; REDDING, S. Technological convergence, R\&D, trade and productivity growth. European Economic Review, n. 49, p. 775-807, 2003.

CAVALLO, M.; LANDRY, A. The Quantitative Role of Capital-Goods Imports in U.S. Growth. American Economic Review, Papers and Proceedings, v. 100, n. 2, May, p. 78-82, 2010.

CHOUDHRI, E.U.; HAKURA, D.S. International Trade and Economic Growth: Exploring the Sectoral Effects for Developing Countries. IMF Staff Papers, v. 47, n. 1, 2000.

ENDERS, W. Applied Econometrics Time Series. New York: John Wiley \& Sons Inc, 2004.

Rats Handbook for Econometric Time Series. New York: John Wiley \& Sons Inc, 1996.

FERREIRA, P.C.; GULLÉN, O.T.C. O impacto da abertura comercial sobre mark-up e produtividade industrial brasileira. Ensaios Economicos da EPGE, Fundação Getúlio Vargas, n. 432, 2004.

FERREIRA, P.C.; ROSSI JR., J.L. New Evidence from Brazil on Trade Liberalization and Productivity Growth. International Economic Review, v. 44, n. 4, 2003.

GILES, J.A.; WILLIAM, C.L. Export-led Growth: A Survey of the Empirical Literature and Some Noncausality Results. Econometrics Working Paper, n. 9, p. 901, 2000.

GONÇALVES, R. et al. A Nova Economia Internacional: uma perspectiva brasileira. Rio de Janeiro: Campus, 1998.

GOSH, K.; SAUNDERS, P.J.; BISWAS, B. An Empirical Investigation of the Relations among Wage Differentials, Productivity Growth, and Trade. Contemporary Economic Policy, v. 20, n. 1, january, p. 83-92, 2002.

HALPERN, L.; KOREN, M.; SZEIDL A. Imports and Productivity. CEPR Discussion Paper, n. 5139, July, 2005. 
HANSEN, H.; JUSELIUS, K. Cats in Rats - Cointegration Analysis of Time Series. USA: Estima, 1995.

HAY, D.A. The Post-1990 Brazilian trade liberalisation and the performance of large manufacturing firms: productivity, market share and profits. The Economic Journal, n. 111, July, p. $620-641,2001$.

HUNG, J.; SALOMON, J.M.; SOWERBY, S. International Trade and US Productivity Research. International Business and Finance, v. 18, p. 1-25, 2004.

JOHNSTON, J.; DINARDO, J. Métodos Econométricos. 4 ed. Amadora, Portugal: McGrawHill, 2001.

KASAHARA, H.; LAPHAM, B.J. Productivity and the decision to import and export: theory and evidence. CESifo Working Paper Series, n. 2.240, March, 2008.

KIM, S.; KIM, J.Y. Growth gains from trade and education. Journal of International Economics, v. 50, n. 2, p. $519-545,2000$.

LAWRENCE, R.Z; WEINSTEIN, D.E. Trade and Growth: Import-led or Export-led? Evidence from Japan and Korea. NBER Working Paper, n. 7.264, 1999.

LIN, C.W.; WONG, K. Economic growth and international trade: the case of Hong Kong. Discussion Papers in Economics at the University of Washington, n. 0080, Department of Economics at the University of Washington, 1997.

LISBOA, M.B.; MENEZES FILHO, N.; SCHOR, A. Os Efeitos da Liberalização Comercial sobre a Produtividade: Competição ou Tecnologia? In: Encontro da Sociedade Brasileira de Econometria, 23, Nova Friburgo. Anais do XXIII Encontro da Sociedade Brasileira de Econometria, 2000.

MUENDLER, M. Trade, Technology, and Productivity: A Study of Brazilian Manufacturers, 1986-1998. UC San Diego: Department of Economics, UCSD. Dísponivel em: http://escholarship.org/uc/item/6m96c2r7, último acesso: 01/2010.

NETTO, C.R.S.; CURADO, M.L. Produtividade do Trabalho, Salários Reais e Desemprego na Indústria de Transformação na Década de 90: Teoria e Evidência. Revista de Economia Contemporânea, Rio de Janeiro, v. 9, n. 3, p. 485-508, 2005.

ROSSI Jr., J.L.; FERREIRA, P.C. Evolução da Produtividade Industrial Brasileira e Abertura Comercial. Texto para Discussão, IPEA, n. 651, Brasília, 1999.

SABÓIA, J.; CARVALHO, P.G.M. Produtividade na Indústria Brasileira-Questões Metodológicas e Análise Empírica. Texto para Discussão, IPEA, n. 504, Brasília, 1997.

SILVA, D. O Impacto da abertura comercial sobre a produtividade da indústria de transformação brasileira. Dissertação de Mestrado em Finanças e Economia Empresarial, EPGE, Fundação Getúlio Vargas, 2004. 


\section{APÊNDICE}

\section{Gráfico 1 - Série PRODHT}

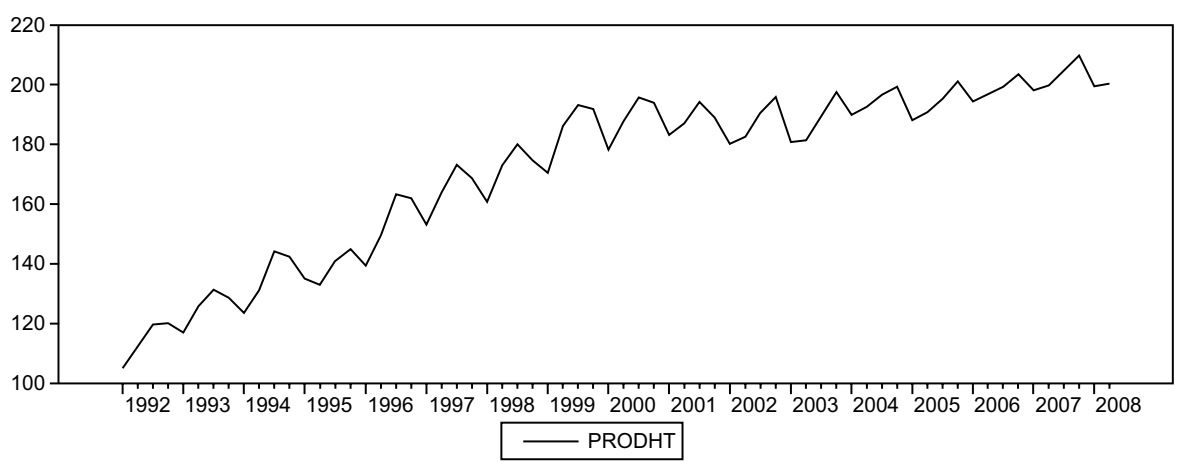

Fonte: Elaboração própria a partir de dados da PIMES (IBGE) e CNI.

\section{Gráfico 2 - Série M}

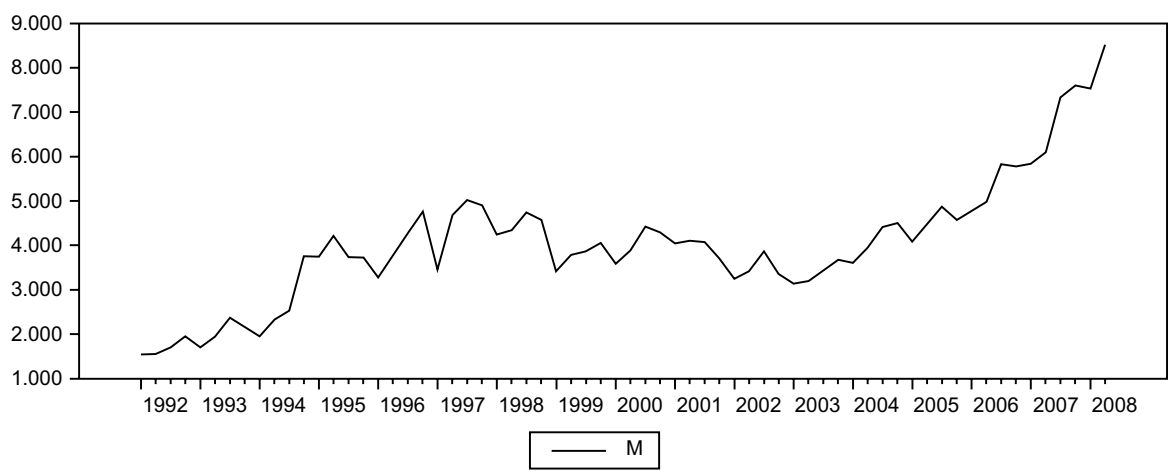

Fonte: Elaboração própria a partir de dados do MDIC e IPEADATA.

\section{Gráfico 3 - Teste beta conhecido - variáveis PRODHT e M}

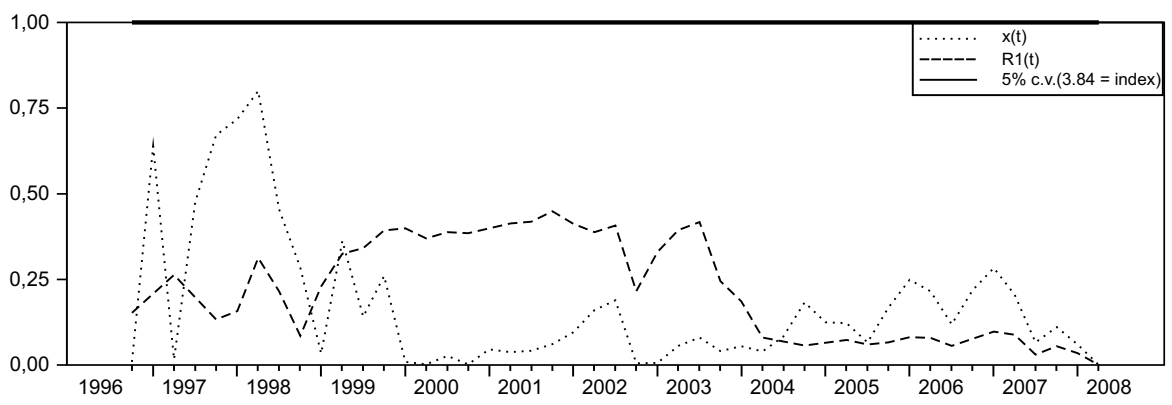

Fonte: Elaboração própria. 
Tabela 7 - Teste ADF- Séries em Nível

\begin{tabular}{l|c|l|c|c}
\hline & No de Defasagens & \multicolumn{1}{c}{ Teste } & Calculado & Tabelado \\
\hline & & Regressão com Constante e Tendência & $-1,5479$ & $-3,41$ \\
\hline PRODHT & 4 & Regressão com Constante e sem Tendência & $-2,13131$ & $-2,86$ \\
\hline & & Regressão sem Constante e sem Tendência & 1,40734 & $-1,95$ \\
\hline & & Regressão com Constante e Tendência & $-0,6998$ & $-3,41$ \\
\hline M & 4 & Regressão com Constante e sem Tendência & 0,28902 & $-2,86$ \\
\hline & & Regressão sem Constante e sem Tendência & 1,52064 & $-1,95$ \\
\hline
\end{tabular}

Fonte: Elaboração própria.

Tabela 8 - Teste ADF - Séries em Primeira Diferença

\begin{tabular}{l|c|l|c|c}
\hline & $\mathbf{N}^{\circ}$ de Defasagens & Teste & Calculado & Tabelado \\
\hline & & Regressão com Constante e Tendência & $-3,37883$ & $-3,41$ \\
\hline \multirow{2}{*}{$\Delta$ PRODHT } & 3 & Regressão com Constante e sem Tendência & - & - \\
\hline & & Regressão sem Constante e sem Tendência & - & - \\
\hline & & Regressão com Constante e Tendência & $-8,75402$ & $-3,41$ \\
\hline $\mathrm{M}$ & 0 & Regressão com Constante e sem Tendência & - & - \\
\hline
\end{tabular}

Fonte: Elaboração própria.

Tabela 9 - Teste HEGY

\begin{tabular}{l|l|c|c|c|c}
\hline & & \multicolumn{2}{|c|}{$\begin{array}{r}\text { Raiz Unitária Não } \\
\text { Sazonal }\end{array}$} & \multicolumn{2}{c}{ Raiz Unitária Sazonal } \\
\hline & & Calculado & Tabelado & Calculado & Tabelado \\
\hline & Regressão com Constante & $-2,374$ & $-2,88$ & 0,723 & 3,08 \\
\hline PRODHT & Regressão com Constante e Dummy Sazonal & $-2,28$ & $-2,95$ & 2,75 & 6,57 \\
\hline & $\begin{array}{l}\text { Regressão com Constante, Dummy Sazonal } \\
\text { e Tendência }\end{array}$ & $-1,458$ & $-3,53$ & 2,722 & 6,6 \\
\hline \multirow{2}{*}{} & Regressão com Constante & 0,517 & $-2,88$ & 2,052 & 3,08 \\
\hline & Regressão com Constante e Dummy Sazonal & 0,304 & $-2,95$ & 9,835 & 6,57 \\
\hline & $\begin{array}{l}\text { Regressão com Constante, Dummy Sazonal } \\
\text { e Tendência }\end{array}$ & $-0,631$ & $-3,53$ & 9,945 & 6,6 \\
\hline
\end{tabular}

Fonte: Elaboração própria. 
Tabela 10 - Teste de Phillips-Perron

\begin{tabular}{l|c|c|c|c}
\hline & \multicolumn{3}{|c|}{ Estatística de Teste } & \multirow{2}{*}{$\begin{array}{c}\text { Valor Crítico } \\
\text { (5\% nível de significância) }\end{array}$} \\
\hline Raiz Unitária & $\begin{array}{c}\text { Quebra Estrutural } \\
\text { de Pulso (DU) }\end{array}$ & $\begin{array}{c}\text { Quebra Estrutural } \\
\text { de Nível (DTb) }\end{array}$ & $-1,55606$ & $-5,59$ \\
\hline PRODHT & $-4,71888$ & 4,63832 & 1,03432 & $-5,59$ \\
\hline M & $-3,27892$ & $-3,43903$ & & \\
\hline
\end{tabular}

Fonte: Elaboração própria.

Tabela 11 - Modelo PRODHT, QXM e TXCR

\begin{tabular}{|c|c|c|c|c|}
\hline \multicolumn{5}{|c|}{ Equação 1 - Variável Dependente: PRODHT } \\
\hline Variáveis Explicativas & Coeficientes & Desvio Padrão & t-student & P-valor \\
\hline D_PRODHT(1) & $-0,13189$ & 0,13760 & $-0,95853$ & 0,34232 \\
\hline D_PRODHT(2) & $-0,46948$ & 0,12196 & $-3,84944$ & 0,00033 \\
\hline D_PRODHT(3) & $-0,09017$ & 0,13377 & $-0,67412$ & 0,50328 \\
\hline D_M(1) & 0,00008 & 0,00133 & 0,05802 & 0,95396 \\
\hline D_M(2) & $-0,00315$ & 0,00130 & $-2,43358$ & 0,01849 \\
\hline D_M(3) & $-0,00158$ & 0,00135 & $-1,17056$ & 0,24722 \\
\hline Constant & 8,85730 & 4,07307 & 2,17460 & 0,03432 \\
\hline DUMMY2 & 8,78790 & 1,91295 & 4,59390 & 0,00003 \\
\hline DUMMY3 & 5,42621 & 2,68678 & 2,01960 & 0,04869 \\
\hline DUMMY4 & 6,57597 & 2,36623 & 2,77909 & 0,00761 \\
\hline $\mathrm{EC} 1\{1\}$ & $-0,08719$ & 0,02506 & $-3,47874$ & 0,00104 \\
\hline \multicolumn{5}{|c|}{ Equação 2 - Variável Dependente: M } \\
\hline Variáveis Explicativas & Coeficientes & Desvio Padrão & t-student & P-valor \\
\hline D_PRODHT(1) & 46,70168 & 14,49005 & 3,22302 & 0,00221 \\
\hline D_PRODHT(2) & 0,66735 & 12,84311 & 0,05196 & 0,95876 \\
\hline D_PRODHT(3) & 21,27851 & 14,08632 & 1,51058 & 0,13707 \\
\hline D_M(1) & $-0,08485$ & 0,14010 & $-0,60569$ & 0,54741 \\
\hline D_M(2) & 0,28030 & 0,13645 & 2,05418 & 0,04510 \\
\hline D_M(3) & 0,11758 & 0,14178 & 0,82932 & 0,41079 \\
\hline Constant & $-1067,78935$ & 428,92016 & $-2,48948$ & 0,01610 \\
\hline DUMMY2 & 1173,39388 & 201,44582 & 5,82486 & 0,00000 \\
\hline DUMMY3 & 899,00836 & 282,93456 & 3,17744 & 0,00252 \\
\hline DUMMY4 & 510,35665 & 249,17896 & 2,04815 & 0,04571 \\
\hline $\mathrm{EC} 1\{1\}$ & 3,13671 & 2,63924 & 1,18849 & 0,24015 \\
\hline
\end{tabular}

Fonte: Elaboração própria. 